\title{
EDITORIAL
}

\section{Directions from a reader}

A few Editorials back I wrote discussing what the contributors expected of a scientific journal such as the British Journal of Nutrition. This resulted in a letter from a regular reader of the Journal from outside the nutritional field suggesting that we should also consider the needs of readers who were not necessarily potential contributors but who read the journal to expand their nutritional knowledge for use in other areas of study. I thought that this raised some interesting points, hence the invited Editorial which follows from Dr F. D. Richardson of the Department of Applied Mathematics in the University of Cape Town, South Africa.

D. A. T. SouthGate

\section{The needs of readers}

Journals are probably the most important means of communication between scientists. They can only perform this function if they attract both authors and readers. Recent journal editorials and professional society newsletters have mentioned that a journal must attract a range of the best research papers in its field. They have also focused on the needs of contributors to have their work published and how authors can assist the process. The needs of readers seem to be ignored. This is unfortunate as it is the readers who cite papers, evaluate the practical implications of the findings and ultimately determine the place of the reported work in the body of knowledge.

Why do readers need primary journals when abstracting journals and electronic scanning services cover most fields of scientific endeavour? As pointed out in an earlier editorial, both of these have limitations due to the keywords published with the original paper. Furthermore, even a well prepared scanning profile misses important papers. Obtaining a copy of a paper in a journal not held by the reader's institutional library may involve considerable delay and expense. The implication is that readers do need access to primary journals. This is confirmed by the observation that one of the busiest sections of a scientific library is that where the current issues of journals are displayed.

Before we can establish the needs of readers of a journal we need to know who those readers are. There is a belief that the readers of a scientific journal are more or less the same people as its potential contributors. While this may be true for some very specialized publications it is almost certainly not true for the British Journal of Nutrition because nutrition lies at the interface of a number of scientific disciplines. In addition, nutrition can be the well understood core that links parts of a larger system together. An example of this is a pastoral economy dependent on semi-arid rangeland. Nutritional science provides information and understanding of the factors that influence the selection, voluntary intake and digestibility of the diet of grazing animals, the amounts of nutrients absorbed and the efficiency of their utilization for production. This is the link between weather, soils and herbage growth on the one hand and the output of animal products on the other. Similarly, 
human protein requirements are the interface between the well-being of pastoralists and the productivity of their herds. Knowledge of nutrition is required to interpret the behaviour of the whole system.

The advent of computers and modelling techniques has increased the need for nutritional information and the potential for its use. A wide range of models are based on concepts and data reported in the nutritional literature. Research models of diet selection, voluntary intake, digestion and metabolism designed to test alternative hypotheses are appropriate for publication in the British Journal of Nutrition. However, a mechanistic model designed to increase understanding of the pastoral system mentioned above would normally be described in a rangeland or agricultural systems journal. Predictive models are developed as an aid to the planning, management and monitoring of, for example, livestock production systems or child feeding programmes. Such models are frequently sophisticated both conceptually and mathematically. Some of them are published in formal scientific and professional journals, others as monographs or policy documents by development agencies and international organizations. The availability of the latter may be restricted to the organization that commissioned the models or its clients.

Apparently there are three groups of readers of the British Journal of Nutrition: (1) research workers who are potential contributors to the Journal; (2) research workers in related fields who are potential contributors to other scientific journals; (3) scientists who are not specifically engaged in research but who need and use nutritional information. The last group is probably of increasing importance. They may be engaged in developing policy, planning projects and acting as consultants to international, state and commercial organizations. Researchers are advised to remember this group when planning investigation. Citation of papers for this purpose could in future have a major impact on research grants.

This brings us to the point: what do readers require of the journal?

Briefly this is accessible, usable and reliable information.

Readers want a journal that attracts a high proportion of the important research papers in their specific and associated fields of interest. It must have a good index and, if possible, a cumulative index as these are valuable aids when searching for information.

The papers must present clearly-reasoned concepts of mechanisms and processes. The data should be suitable for estimating the parameters of models or for their validation. The exact conditions of an experiment must be described. Validation of a model involves the repetition of experiments as computer simulations and other research workers need to know how the findings related to their own work.

Readers require a journal that employs rigorous standards of peer review so that they can use the published material with confidence.

The references in a paper are a source of additional information. However, their use can be very frustrating when reference is made to material that is not readily available. There are three common problems: (1) references to theses: has the information been published in a journal or submitted? (2) cascaded references: the reference cited must be to the publication where the material was actually published. Readers should not need to order from a distant library copies of four papers in succession before obtaining a description of the method of assay used, (3) conference proceedings: will the relevant information be published elsewhere?

Sometimes readers require more information than can be presented in a published paper. An Editorial last year mentioned the possibility of archiving the data on which the results presented in papers are based. Editorial boards and professional societies may be the appropriate bodies to formulate and monitor rules and ethical standards for access to and use of these data. For example, if a substantial body of archival data is used in a new paper, 
mere reference to unpublished results could be inadequate recognition of the contribution of the original author.

When authors choose a journal to receive their papers, they would be well advised to identify the readers they wish to target and then select the publication that meets the needs of those readers.

F. D. RICHARDSON 